\title{
CpG binding protein (CFP1) occupies open chromatin regions of active genes, including enhancers and non-CpG islands
}

Louie N. van de Lagemaat ${ }^{1}$ (D, Maria Flenley ${ }^{2}$, Magnus D. Lynch 2,3,7, David Garrick ${ }^{4}$, Simon R. Tomlinson ${ }^{5}$, Kamil R. Kranc ${ }^{5,6}$ and Douglas Vernimmen ${ }^{1 *}$ (D)

\begin{abstract}
Background: The mechanism by which protein complexes interact to regulate the deposition of post-translational modifications of histones remains poorly understood. This is particularly important at regulatory regions, such as CpG islands (CGIs), which are known to recruit Trithorax ( $\operatorname{Tr} x G)$ and Polycomb group proteins. The CxxC zinc finger protein 1 (CFP1, also known as CGBP) is a subunit of the TrXG SET1 protein complex, a major catalyst of trimethylation of H3K4 (H3K4me3).
\end{abstract}

Results: Here, we used ChIP followed by high-throughput sequencing (ChIP-seq) to analyse genomic occupancy of CFP1 in two human haematopoietic cell types. We demonstrate that CFP1 occupies CGIs associated with active transcription start sites (TSSs), and is mutually exclusive with H3K27 trimethylation (H3K27me3), a marker of polycomb repressive complex 2. Strikingly, rather than being restricted to active CGI TSSS, CFP1 also occupies a substantial fraction of active non-CGI TSSs and enhancers of transcribed genes. However, relative to other TrxG subunits, CFP1 was specialised to TSSs. Finally, we found enrichment of CpG-containing DNA motifs in CFP1 peaks at CGl promoters.

Conclusions: We found that CFP1 is not solely recruited to CpG islands as it was originally defined, but also other regions including non- $\mathrm{CpG}$ island promoters and enhancers.

Keywords: CFP1, CpG islands, Trithorax group proteins, Epigenetics, Enhancers

\section{Background}

In vertebrate genomes, $\mathrm{CpG}$ dinucleotides are relatively depleted, except in specific DNA regions with a high density of this dinucleotide. These regions are known as CpG islands (CGIs) and consist of short ( $1000 \mathrm{bp})$ interspersed CpG-rich and predominantly unmethylated DNA sequences [1], which are associated with transcriptionally permissive chromatin state [2]. Interestingly, the human genome harbours roughly twofold more annotated CGIs than the mouse genome $(27,000$ versus 15,000$)$ [3]. CGIs were originally identified in promoters of housekeeping genes and associated with $\mathrm{H} 3 \mathrm{~K} 4 \mathrm{me} 3$ independent of gene

\footnotetext{
*Correspondence: douglas.vernimmen@roslin.ed.ac.uk

${ }^{1}$ The Roslin Institute and Royal (Dick) School of Veterinary Studies, University of Edinburgh, Easter Bush, Midlothian EH25 9RG, UK Full list of author information is available at the end of the article
}

activity [1]. However, it is now recognised that CGIs are also found in about half the promoters of developmentally regulated/tissue-specific genes [4]. In this group of CGIs, repressive polycomb group (PcG) complexes act to block transcription in inappropriate lineages or at nonexpressing stages during the differentiation programme. It has been hypothesised that CGIs at developmentally regulated genes of higher organisms may be relics of ancestral CGIs which have been differentially maintained during evolution, for example, in humans and rodents [3-8]. Thus, at present, the functional significance of these CGIs for the correct regulation of developmentally regulated genes is poorly understood. A good example of this is the well-studied $\alpha$-globin locus. Mouse $\alpha$-globin genes, which lack an annotated CGI, exhibit tightly regulated tissue and developmental stage-specific regulation 
similar to that observed for the human $\alpha$-globin genes, which contain a CGI and are regulated by PcG [9].

Several proteins have been described in mammals that bind specifically to CGIs. These proteins contain the $\mathrm{CxxC}$ zinc finger (ZF-CxxC) domain and include CFP1, MLL1, MLL2, KDM2A, KDM2B, TET1 and TET3 [10]. Whilst MLL1/2 also have inherent histone lysine N-methyltransferase activity, CFP1 does not, instead forming complexes with SET1A and SET1B methyltransferases, TrxG subunits of the SET1 family [11]. CFP1-SET1A/B complexes have been implicated in development $[11,12]$ and are known to target a proportion of H3K4 methylation to CGIs [12, 13]. CFP1 binds unmethylated CpG-rich sequence [14], and is associated with $\mathrm{H} 3 \mathrm{~K} 4$ trimethylation at transcription start sites (TSSs) irrespective of transcription level [15, 16], although recent evidence showed that it preferentially binds CGIs of actively transcribed genes [17]. Two other TrxG complexes, MLL3 and MLL4, lack ZF-CxxC domains [10] and have been suggested to be involved in deposition of H3K4me1 at enhancer elements [18-20].

Multiple phenotypes have been documented for CFP1 deficiency, both at the cellular and organismal level. CFP1 is required for development [21], differentiation of embryonic stem (ES) cells [22] and haematopoiesis [23-25]. CFP1 binds to unmethylated CGIs in mouse ES cells $[15,17]$ and mouse brain tissues [16], and knockout and knockdown of CFP1 in mouse ES cells and NIH3T3 fibroblasts result in reduction of $\mathrm{H} 3 \mathrm{~K} 4 \mathrm{me} 3$ levels at CGIs $[16,17]$. Mouse ES cells lacking CFP1 are viable but are unable to differentiate $[22,26]$. In these cells, H3K4me3 was found to be mainly reduced at highly expressed genes, while the levels at other CGIs such as bivalent domains were largely unchanged [15]. These observations may be explained by the above-mentioned CFP1 preference for actively transcribed genes and the finding that MLL2 (rather than SET1) complexes are largely responsible for initial deposition of $\mathrm{H} 3 \mathrm{~K} 4 \mathrm{me} 3$ at bivalent domains [27, 28].

Here we have analysed the genome-wide occupancy of CFP1 in two divergent human haematopoietic cell types: erythroid (ERY) and EBV-transformed B-lymphoid (EBV) cells. Previous studies using chromatin immunoprecipitation (ChIP) have been restricted to a couple of available antibodies with moderate signal, which consequently limited the sensitivity of the analysis $[15,29]$. Here, using a non-commercial, high-affinity antibody [30], our analysis reveals that the presence of CFP1 at accessible CGI TSSs is associated with Pol II binding and gene expression. Moreover, H3K27me3, which is a mark of PcG (PRC2) activity, and binding of CFP1 are mutually exclusive at TSSs. Unexpectedly, CFP1 is also recruited to a substantial fraction of non-CGI-associated TSSs and active enhancer elements. In comparison with other TrxG complex subunits, CFP1 is specialised to active TSSs. Finally, our analysis revealed enrichment of CpG dinucleotide-containing motifs in CFP1 peaks at CGIs.

\section{Results \\ CFP1 primarily binds at active CGI TSSs and is associated with transcription}

We previously reported an association of CFP1 binding with transcription at the $\alpha$-globin locus, using a mouse model in which the mouse $\alpha$-globin locus was replaced by the human locus $[29,31]$. In this "humanised" mouse model, the epigenetic regulation of the human locus in mouse erythroblasts mirrors that observed in human primary erythroblasts, including recruitment of PcG when the gene is silenced [29]. In these humanised mouse erythroid cells, the targeted deletion of a key human $\alpha$-globin enhancer (MCS-R2, also known as HS-40) results in a strong reduction in $\alpha$-globin transcription $[31,32]$. This is associated with the maintenance of PcG binding [29] and an impairment of CFP1 recruitment to the human $\alpha$-globin promoter [29]. Similarly, in human primary erythroid and lymphoid (EBV-transformed) cell types, where this locus is active and inactive, respectively, CFP1 is recruited to the CGI spanning the $\alpha$-globin gene only in erythroid cells [29]. Experiments with a robust, non-commercial CFP1 antibody [30] confirmed these results in primary cells (Additional file 1: Fig. S1A) and in the humanised mouse model (Additional file 1: Fig. S1B). ChIP signals derived from this antibody were several fold stronger than a comparable commercial CFP1 antibody (Additional file 1: Fig. S1C). The non-commercial antibody was tenfold more sensitive (14,872 peaks, as described below, versus 1434 in erythroid cells), and in 1102 locations where the peaks overlapped it showed a median 2.3 fold higher enrichment of reads in peaks given a similar number of ChIP-seq reads and the same input data. Given this tenfold greater sensitivity and 2.3fold greater specificity, it was the primary antibody used in this study.

To identify genomic regions recruiting CFP1, we performed ChIP-seq in primary human lymphoid and erythroid cell types (biological replicates from Fig. S1A, Additional file 1). As shown in Fig. 1a, heatmaps of genomic TSSs and CGIs showed that these regions were strongly enriched in CFP1 binding. As shown in Fig. 1b, genomic CFP1 localisation was remarkably similar in both cell types, with a large number of peaks located at TSSs with annotated CGIs: $62.2 \%(9251 / 14,872)$ and $42.5 \%(9055 / 21,301)$ of genome-wide CFP1 summits were associated with CGI promoters in erythroid and lymphoid cells, respectively. Amongst non-CGI genomic regions of CFP1 binding, TSSs were less prominent 
(Fig. 1b). As shown in Fig. S1D (Additional file 1), CGI and non-CGI TSSs differed strongly in CpG content within a 1-kb window centred on TSSs. CpG content of representative CGI and non-CGI loci considered hereafter are graphed in Fig. S1D (Additional file 1) .

As shown in Fig. 1a, CFP1-bound TSSs were depleted in H3K27me3 and H3K4me1 histone marks, whilst being strongly enriched in H3K4me3. The association between CFP1 and H3K4me3 could either reflect deposition of H3K4me3 by CFP1-SET1A/B complexes, or binding to pre-existing H3K4me3, deposited by other TrxG complexes [33], through the PHD finger domain of CFP1 [17]. Accordingly, we found strong association between peaks of CFP1 and H3K4me3 in both erythroid and lymphoid cell types, both qualitatively at the level of heatmaps (Fig. 1a) and quantitatively in peak overlaps (Fig. 1c). Indeed, the fraction of CFP1 peaks overlapped with H3K4me3 was nearly $100 \%$ when the alternate peak finder, MACS2, was used (Additional file 1: Fig. S2).

In contrast to H3K4me3 and CFP1, little if any colocalisation was observed between CFP1 and H3K27me3. To further characterise depletion of H3K27me3 (a histone mark associated with PcG binding) in CFP1-bound regions, we plotted averaged read depth in a 2-kb window surrounding TSSs for CFP1 versus H3K27me3. As shown in Fig. 1d, TSSs showed mutual exclusivity between H3K27me3 and CFP1 occupancy, with marked preference for binding of either CFP1 or PRC2 (H3K27me3), or neither, but not both; this was observed consistently in erythroid and lymphoid cell types. This result demonstrates that mutual exclusivity of CFP1 and the H3K27me3 mark at TSSs, which was first described in the $\alpha$-globin locus [29], is a general genome-wide mechanism in disparate cell types.

\section{Binding of CFP1 to housekeeping and tissue-specific gene TSSs}

We next assessed CFP1 occupancy at TSSs by gene class, specifically housekeeping genes and developmentally regulated/tissue-specific genes (Fig. 2 and Additional file 1: Fig. S3). Both the $\alpha$-globin locus in erythroblasts (Fig. 2a, left) and IRF4 locus in lymphoid cells (Fig. 2a, right) exhibit lineage-specific binding of CFP1. Housekeeping genes such as ACTB and LUC7L (located just downstream of the $\alpha$-globin locus) showed binding in both cell types (Fig. 2b). The CGI promoter of RHBDF1, which is not expressed in either cell type, was marked by H3K27me3 in both erythroid and lymphoid cell types (Additional file 1: Fig. S3C), but was not bound by CFP1 (Fig. 2c).

We then extended our analysis to genome-wide sets of housekeeping and developmentally regulated/tissue-specific genes. First, we used the Illumina Body Map RNAseq data set of 16 human tissues to identify 5354 genes with similar expression across most or all of these tissues and termed these housekeeping genes (Fig. 2d; see "Methods"). Another 7150 genes with low expression in nine or more of 16 tissues were termed candidate tissuespecific genes. RNA-seq data sets from erythroblasts and EBV-transformed lymphoid cells were then used to identify candidate tissue-specific genes with elevated expression in these cell types (412 genes in erythroid and 658 in lymphoid cells; see "Methods"). Interestingly, CXXC1 mRNA, which encodes CFP1 protein, was denominated a housekeeping gene, with mean RPKM (reads per kilobase of transcript per million mapped reads) of 5.8 ( $\pm 3.1 \mathrm{SD})$. Public expression data sets for EBV and ERY cells showed overexpression of CXXC1 in EBV $(\mathrm{RPKM}=50.6)$ and reduced expression in ERY $(\mathrm{RPKM}=0.62)$. This mRNA expression difference corresponded to approximately twofold higher expression at the protein level in lymphoid cells (Additional file 1: Fig. S4).

To address the confounding effect of alternate TSSs of transcribed genes, some with or without CGIs, and some transcribed or not, we next limited our analysis to putatively expressed TSSs. These were identified by accessible chromatin, which was defined by the presence of $1 x$-normalised/input-subtracted ATAC (Assay for TransposaseAccessible Chromatin) signal $>10$ within $1 \mathrm{~kb}$ of the TSS. Most putatively expressed TSSs regardless of gene class were marked by CGIs (defined by a distance $<1 \mathrm{~kb}$; green dotted lines, Fig. 2d). However, a larger proportion of housekeeping gene TSSs were marked by a CGI $(94.7 \%$ in erythroid and $96.8 \%$ in lymphoid cells), compared to

\footnotetext{
(See figure on next page.)

Fig. 1 Genomic distribution of CFP1 relative to CpG islands, transcription start sites (TSSs) and marks of active and repressed chromatin. a Heatmap plots of human genomic 6-kb regions centred on TSSs (top) and CGIs (bottom) in erythroblasts (ERY) and Epstein-Barr virus transformed lymphoblast (EBV) cells. TSSs sorted in order of CFP1 coverage; CGIs sorted in order of size. Sequencing read depths shown for CFP1, H3K27me3, H3K27ac, H3K4me1, H3K4me3, DNase-seq, ATAC-seq and Pol Il are net coverage after normalisation to 1x genome-wide and subtraction of an input data set similarly normalised. Gene expression ( $\log _{2}$ RPKM) is shown to the right for TSSs in both cell types. $\mathbf{b}$ Location of CFP1 summits allocated in order of CGI TSS ( $<1 \mathrm{~kb}$ ), CGI genic, CGl intergenic, (non-CGI) TSS ( $<1 \mathrm{~kb}$ ), (non-CGl) genic and (non-CGl) intergenic. ERY, top; and EBV, bottom. $\mathbf{c}$ Venn diagrams showing CFP1 peaks within 1-kb of TSSs are strongly associated with H3K4me3 histone mark and poorly associated with H3K27me3 repressive histone mark. Cell types are ERY (upper) and EBV (lower). Public data sets: * NCBI GEO GSE36985, ** NCBI GEO GSE50893. d Mutual exclusivity of CFP1 and the H3K27me3 mark in ERY (upper) and EBV (lower) cell types
} 


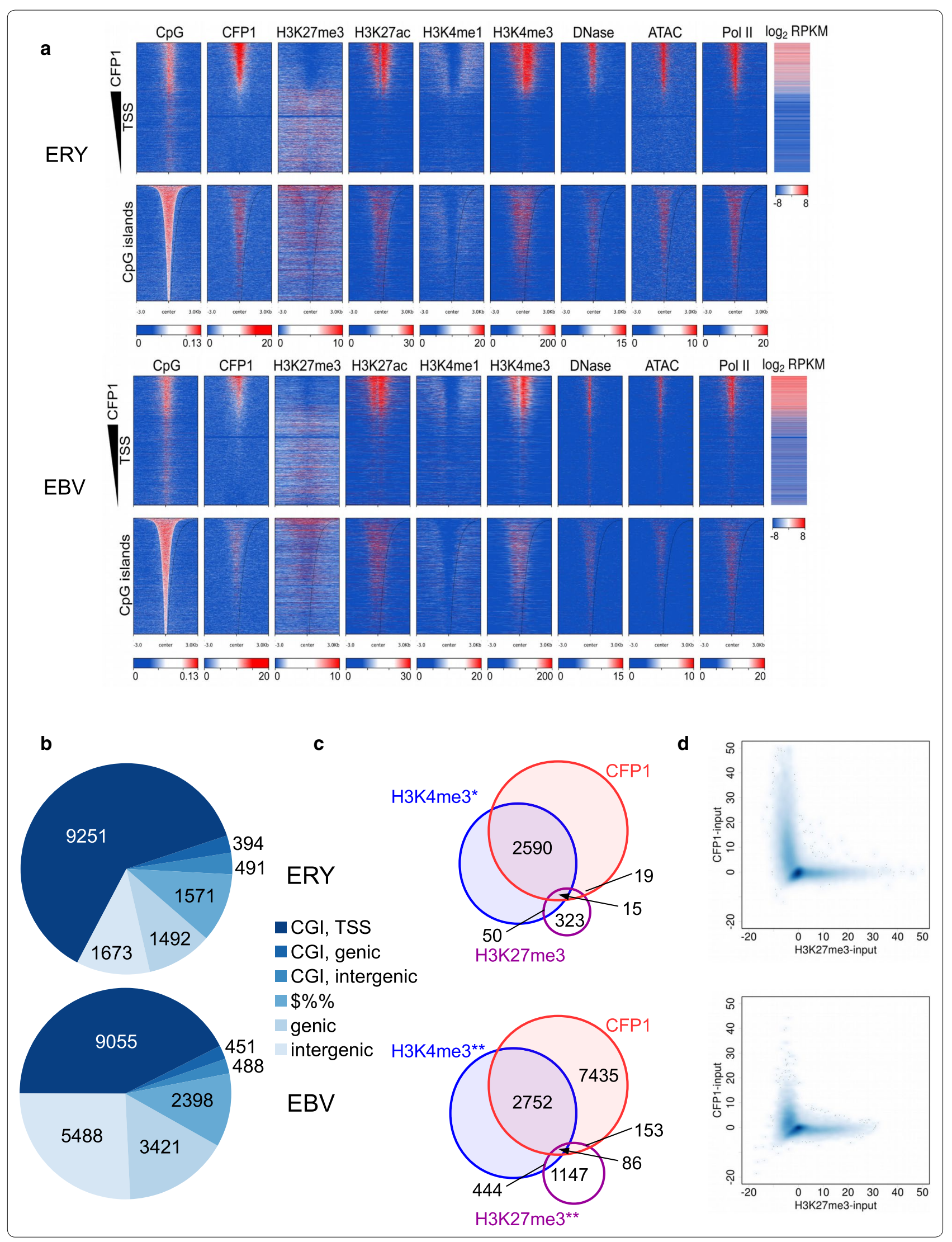




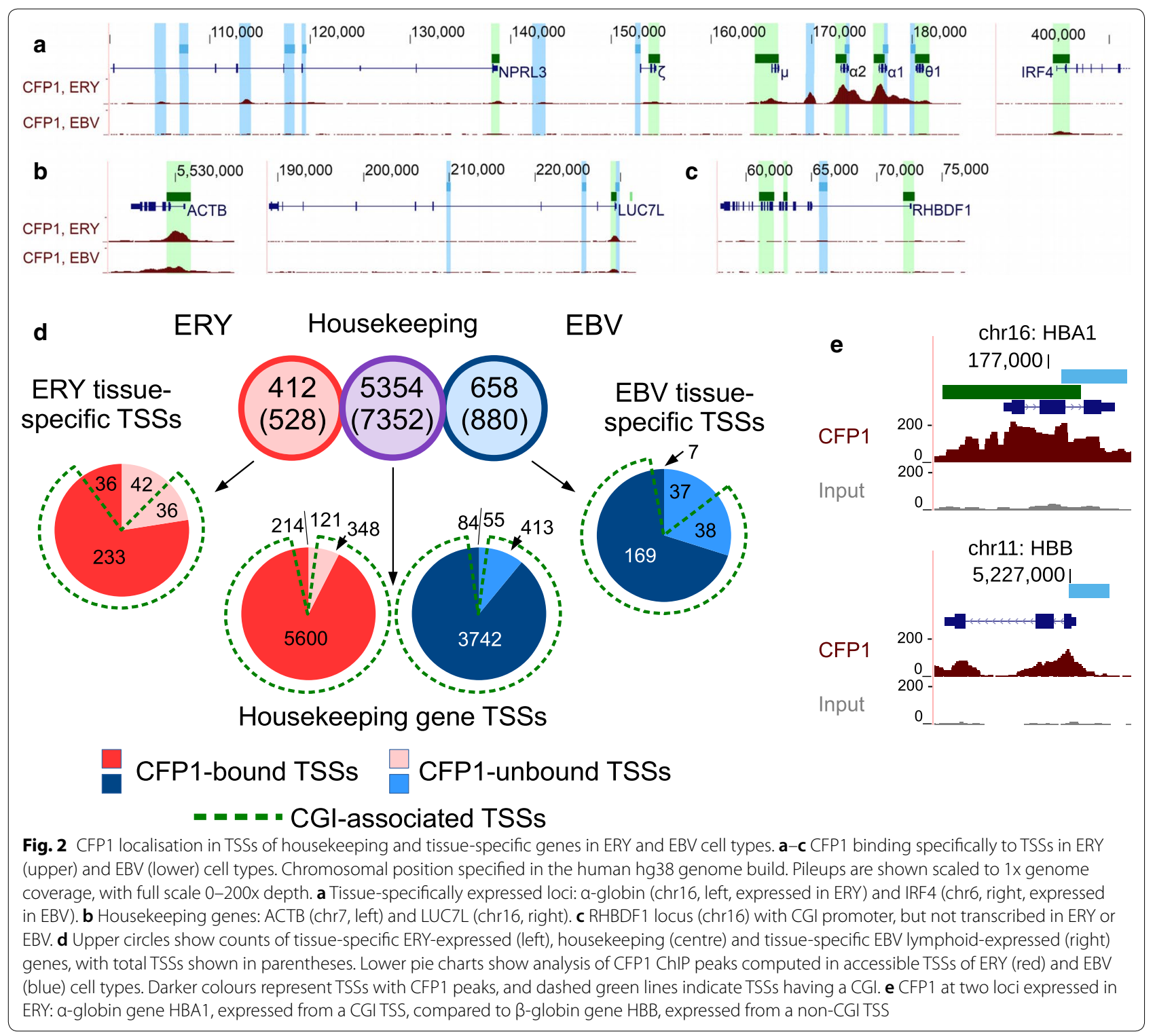

TSSs of tissue-specific genes $77.5 \%$ in erythroid and $82.5 \%$ in lymphoid cells). CGIs accounted for most of the CFP1 occupancy at TSSs, an observation recapitulated in both gene classes and both cell types, and CFP1 peaks were biased for CGI TSSs in all gene classes, as expected $\left(\mathrm{OR} \geq 5.9, p \leq 1.6 \times 10^{-12}\right.$, Additional file 1 : Table S1). Notably, though, housekeeping genes showed increased frequency of CFP1 peaks compared to tissuespecific genes, and this was true in both cell types in both CGI and non-CGI TSSs (Additional file 1: Table S2). Taken together, these findings reinforce the observation that CFP1 occupancy marks active CpG-rich TSSs of both housekeeping and tissue-specific genes, but also reveal that CFP1 is preferentially associated with TSSs of broadly expressed genes whether or not they meet strict criteria for housekeeping genes (see "Methods").

\section{CFP1 binds to non-CGI regions associated with transcription}

Remarkably, although we observed significant CFP1 bias for CGI TSSs, our data also revealed that CFP1 was bound to a proportion of accessible non-CGI TSSs: for example, 36/78 (46\%) and 7/44 (16\%) tissue-specific TSSs in ERY and EBV, respectively (Fig. 2d). More than half of non-CGI housekeeping TSSs were occupied by CFP1, consisting of 214/335 (64\%) TSSs in ERY and 84/139 (60\%) TSSs in EBV (Fig. 2d). In addition to expressed non-CGI TSSs, we found the vast majority 
of annotated, expressed CGI TSSs occupied by CFP1, including 233/269 (86\%) tissue-specific and 5600/5948 (94\%) housekeeping TSSs in ERY. Low CpG density, however, was not necessarily correlated with a lack of CFP1 binding, as exemplified by the well-characterised $\beta$-globin gene HBB. The HBB locus is CpG-poor (Additional file 1: Fig. S1D), but shows similar CFP1 ChIP signal intensity in erythroid cells to that observed at $\alpha$-globin gene promoters (Fig. 2e). Experiments using the previously described CFP1 antibody from Abcam confirmed these results, albeit with weaker ChIP signal (Additional file 1: Fig. S5). These findings call into question the previously reported CGI specificity for this protein [14] and raise the possibility that the recruitment of CFP1 at non-CGI sites may occur by proteinprotein interactions with pre-existing H3K4me3, due to the reader properties of the plant homeodomain (PHD) of CFP1 [17, 26, 33].

\section{Coincident CFP1 and Pol II binding at accessible, expressed TSSs}

We next examined more closely the relationship between CFP1, Pol II and expression level using public RNA-seq data sets. We limited our analysis to accessible TSSs (defined by overlap with a $1 x$-normalised, input-subtracted ATAC-seq signal $>10$ ); this accessibility makes these strong candidate TSSs for the observed expression (Fig. 3a, b). Accessible TSSs of expressed genes in both cell types were occupied with CFP1, regardless of the presence of a CGI (Fig. 3c-f). A few accessible TSSs appeared non-expressed in the available public erythroid data set (GSE74246) while displaying some CFP1 binding in our independent data set (bottom of Fig. 3c, d). We considered it most likely that these are small artefacts due to variability between RNA-seq data sets. Putatively accessible, expressed TSSs were almost universally marked by Pol II (Fig. 3g-j); however, strength of Pol II

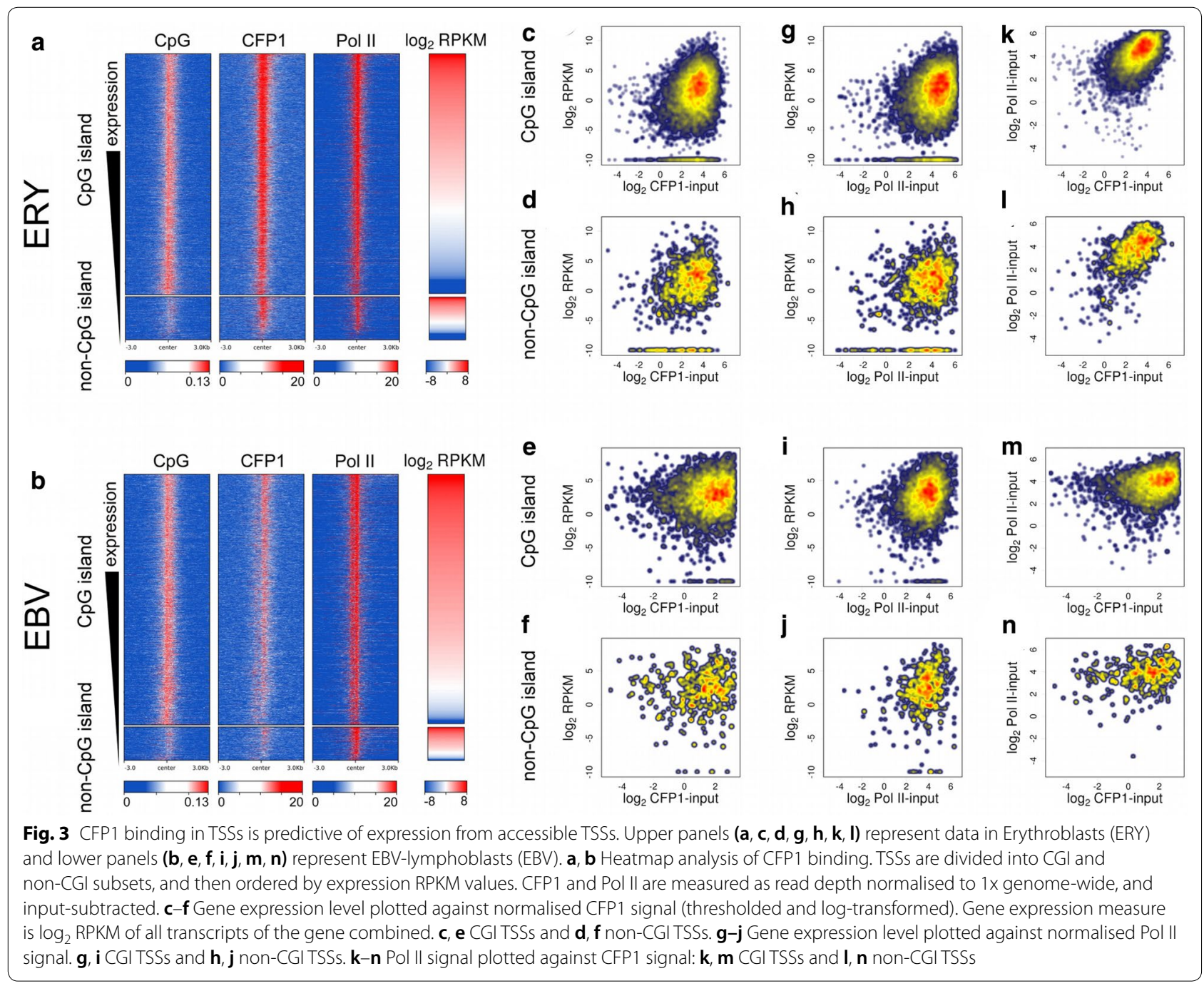


ChIP signals was only poorly correlated with expression, in line with the observations of others [34]. Plotting Pol II versus CFP1 occupancy at accessible TSSs (Fig. $3 k-n)$, we found that TSSs with increased Pol II intensity also exhibited generally increased CFP1 intensity. The correlation between Pol II and CFP1 intensity at these loci was weak in EBV cells (Pearson $R=0.28, p<10^{-200}$, and $R=0.26$, $p=1.4 \times 10^{-12}$, in CGI and non-CGI TSSs, respectively); however, this correlation was visually identifiable in ERY (Pearson $R=0.61, p<10^{-200}$, and $R=0.71, p<10^{-200}$, in CGI and non-CGI TSSs, respectively). Within the limits of public data sets, these results demonstrate a weak positive relationship between Pol II and CFP1 at accessible, transcribed TSSs.

\section{Occupancy of CFP1 at enhancers}

Unmethylated CpG dinucleotides at TSSs are known to recruit CFP1 through its ZF-CxxC domain [14]. However, recent studies have demonstrated that, in addition, CFP1 can bind all three H3K4 methylation states with varying affinity via its PHD zinc finger domain [17, 26, 33]. Frequent localisation of $\mathrm{H} 3 \mathrm{~K} 4 \mathrm{me} 1$ at enhancers could therefore predict the presence of CFP1 at enhancers.

To address localisation of CFP1 at enhancers, putative enhancers were defined by open chromatin (ATACseq) and the presence of Pol II in non-TSS regions (Fig. 4a, see "Methods"). Elevated DNase hypersensitivity, strong bimodal H3K4me1 signal and H3K27ac surrounding these loci confirmed that these sites are likely active regulatory regions. The presence of little or no H3K4me3 and separation from known TSSs rules out a role for these sites as alternative promoters. The elevated ATAC-seq signal in these regions was largely tissue specific, with 10,548 and 3956 putative enhancers identified in erythroid and lymphoid cells, respectively; only 480 overlaps were observed between these sets of putative enhancers. It should be noted that these putative enhancers represent only a stringent subset with an arbitrarily high ATAC-seq signal, and thus they represent the bestsupported candidate enhancers based on the data sets available.

Elevated CFP1 signal was observed at putative enhancers in both cell types, and heatmap evidence suggested qualitative association between CFP1 and signatures of open chromatin (elevated ATAC-seq, DNase-seq, H3K27ac and H3K4me1 signals, Fig. 4a). Indeed, averaged in 2-kb windows around putative enhancers, putative enhancers with above-median ATAC-seq and DNase-seq signals showed elevated CFP1 signal $\left(p<10^{-200}, t\right.$ test, both cell types); this, however, was less intense than in accessible TSSs (Fig. 4b). Furthermore, the correlation of CFP1 signal with chromatin accessibility signals was poorer in enhancers than in TSSs for both cell types (Fig. 4a, c). Nonetheless, CFP1 signal intensity was high at putative enhancers associated with a CpG island. Furthermore, intergenic CFP1 peaks (Fig. 1b) showed strong, non-random overlap with our set of putative enhancers. In erythroid cells, 444/1673 CFP1 peaks were colocalised with putative enhancers, which cover $0.16 \%$ of the genome (166-fold overrepresented, $p<10^{-200}$, binomial test); in lymphoid cells a weaker but specific overrepresentation was noted, with 159/5488 intragenic CFP1 peaks colocalising with this stringently defined set of putative enhancers (18.1-fold overrepresented, $p=1.5 \times 10^{-137}$ ). These findings demonstrate the presence of CFP1 at enhancers and demonstrate that its association with enhancers is specific.

\section{CFP1 colocalises with members of the SET1 complexes}

The chromatin-binding properties of CFP1, aided by a SET1 subunit, anchor SET1A/B complexes in accessible chromatin [12, 13, 17, 26, 35], and these SET1A/B-along with other TrxG-complexes are responsible for H3K4 methylation in accessible chromatin $[13,18-20,27,28$, 36-38]. To investigate this, we performed additional ChIP-seq experiments in erythroid cells. We examined genomic occupancies of SET1A (SETD1A) and Host Cell Factor 1 (HCF1/HCFC1), subunits of the SET1A complexes (Fig. 5, Additional file 1: Fig. S6), and RBBP5, a core subunit of all TrxG complexes [11,39].

To probe the composition of these complexes, we first asked to what extent observed peaks of CFP1, SET1A, HCF1 and RBBP5 were consistent with known composition of SET1A complexes. Upon examination of these ChIP peak overlaps within a high-confidence subset of peak regions (Fig. 5c, see "Methods"), three observations supported the concept that these proteins are subunits of the same complex. First, we observed strong colocalisation between SET1A and CFP1 such that 92.9\% (4266/4594) of SET1A peaks were colocalised with $59.4 \%$ of the 7179 CFP1 peaks (red outline, Fig. 5c). This observation is consistent with a role for CFP1 as a

(See figure on next page.)

Fig. 4 CFP1 binding and histone methylation marks in putative enhancers and comparison to TSSs. a Loci sorted by H3K4me1 signal in 6-kb regions surrounding putative enhancers. Upper panels, ERY; lower, EBV. Note that all data sets, other than CFP1, differ by source between ERY and EBV, which explains prominent differences in signal intensities, for example, of H3K27ac and H3K4me1. b Quantitative comparison of signal intensity in TSSs and putative enhancers, above and below median accessibility (as determined by ATAC-seq data), with cell types indicated. c Plots of ChIP signals for erythroid (left) and lymphoid cells (right), in putative enhancers (lower left graphs) and TSSs (upper right graphs) 

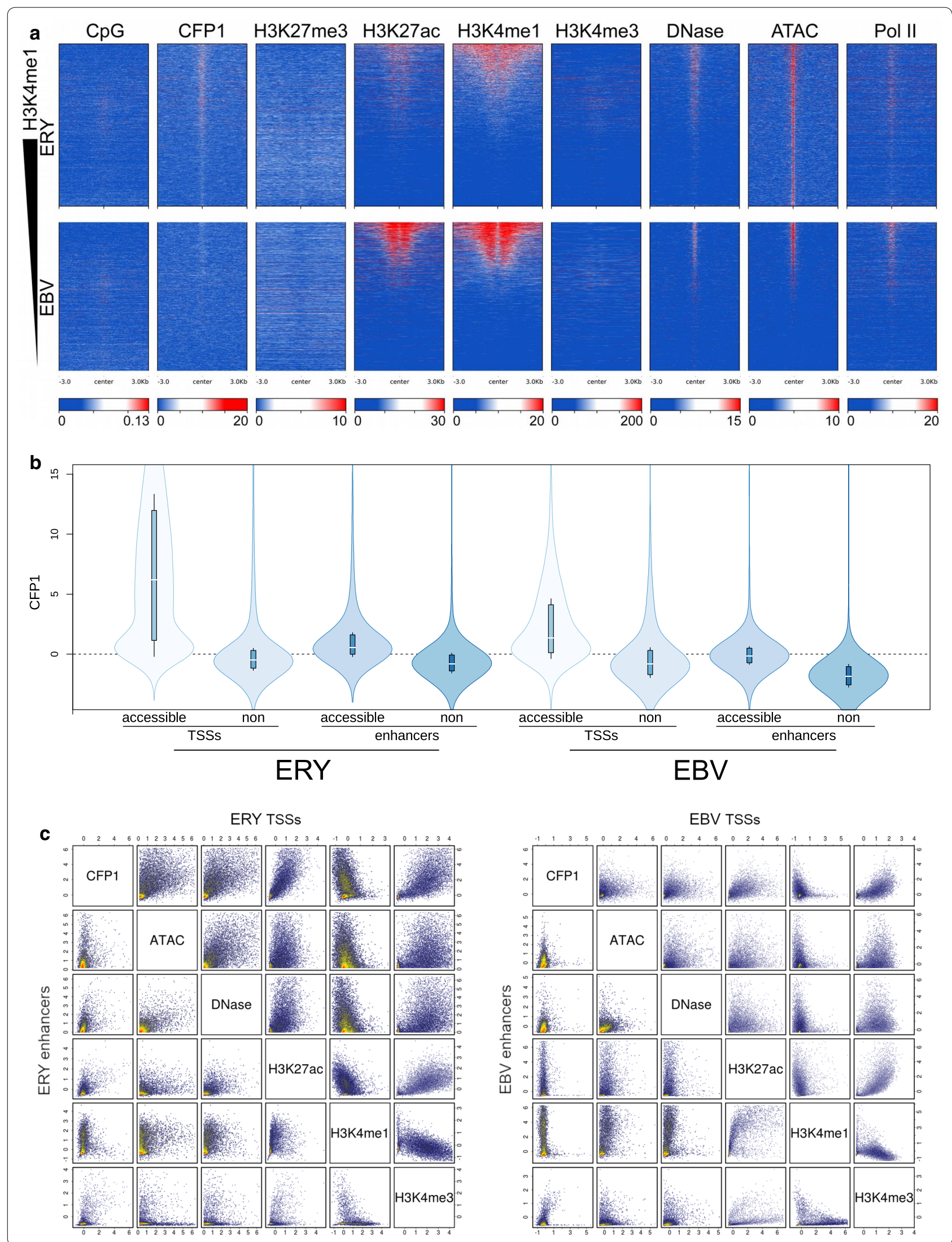
subunit recruiting SET1A and other proteins to bind to DNA. Furthermore it is consistent with the known difference between SET1A and SET1B localisation [38]: excess CFP1 peaks likely are accounted for by SET1B complexes. Secondly, we noted that $90.9 \%$ (6528/7179) of CFP1 peaks were colocalised with RBBP5 (blue outline, Fig. 5c), which is known to form part of a catalytic ASH2L-RBBP5 heterodimer that activates methyltransferase activity in TrxG complexes [36]. This observation suggests a near-complete association of genome-bound CFP1 with RBBP5. Thirdly, we found that a large majority $(85.0 \%, 2047 / 2408)$ of HCF1 peaks were colocalised with CFP1, SET1A and RBBP5 (bold font, Fig. 5c). This and the fact that HCF1 colocalisation accounted for a substantial fraction of these three other peak-sets is consistent with CFP1, HCF1 and RBBP5 being obligate members of SET1A complexes. Taken together, this analysis shows that in regions of high-confidence ChIP signals: (1) there is intimate and specific association between SET1A complex components; (2) genomic SET1A and HCF1 are largely or entirely colocalised with CFP1; and (3) the vast majority of genome-bound CFP1 is associated with RBBP5.

\section{CFP1-SET1A complexes are specialised to TSSs relative to other TrxG complexes}

Notably, the above analysis of SET1A complex subunits showed that RBBP5 had a small but substantial fraction of remaining peaks not colocalised with these subunits, suggestive of additional genomic roles for this protein. Therefore, in an expanded analysis, we considered colocalised SET1A and CFP1 (CFP1-SET1A) as representative of SET1A complexes, Menin as a representative of MLL1/2 complexes and UTX as a representative of MLL3/4 complexes (Additional file 1: Fig. S7). This analysis demonstrated that the excess of RBBP5 peak regions unaccounted for by CFP1-SET1A was accounted for by representatives of the MLL1/2 and MLL3/4 complexes. It also showed that genomic occupancy of HCF1 was almost completely accounted for by overlaps with CFP1SET1A and Menin, which are representatives of SET1 and MLL1/2, respectively. These observations support the view that CFP1, SET1A, HCF1, Menin, UTX and RBBP5 represent a set of proteins whose subunit interactions define compositionally distinct complexes that nevertheless overlap in their genomic occupancy.

We next asked if genomic occupancy of CFP1-SET1A complexes was specialised relative to other TrxG complexes. Specifically, we hypothesised that CFP1-SET1A complexes could differ from other TrxG complexes in their localisation to genomic TSSs and putative enhancers defined earlier. Removing from consideration the small numbers of exclusive CFP1-SET1A $(n=9)$, Menin $(n=583)$ and UTX $(n=507)$ peak regions not colocalising with other TrxG complex subunits (Additional file 1: Fig. S7), we found evidence for substantial site specialisation by the various TrxG complexes (Fig. 5d). CFPSET1A, Menin and UTX peaks were allocated similarly (approximately 5\%) to non-CGI TSSs. In CGI TSSs, all were present at high but differing proportions: $86 \%$ of CFP1-SET1A peaks, representing SET1A complexes, were allocated to CGI TSSs, compared to $74 \%$ of Menin peaks, representing MLL1/2 complexes, and $51 \%$ of UTX peaks, representing MLL3/4 complexes. In our previously defined enhancers, we found the opposite, with putative enhancers representing $6 \%$ of CFP1-SET1A peaks, $9 \%$ of Menin (MLL1/2) peaks and 15\% of UTX (MLL3/4) peaks. In other non-TSS sites of colocalised ChIP peaks, where our conservative criteria did not detect active chromatin, the pattern at enhancers was recapitulated, suggesting that many of these sites act as enhancers. Interestingly, 1409 loci (Additional file 1: Fig. S7) that were external to CFP1-SET1A peaks nevertheless harboured UTX, RBBP5 and Menin (a member of MLL1/2 complexes). Approximately half of these $(n=672)$ were at TSSs. MLL2 is known to deposit H3K4me3 to establish bivalent domains $[27,28,40]$, and UTX is a demethylase known to remove repressive H3K27me3 marks [41]. Finding these proteins colocalised is consistent with physical interaction of UTX with MLL2 complex, a phenomenon postulated from co-immunoprecipitation experiments in differentiated cell lines [40]. Taken together, this analysis of regions of high-confidence ChIP signals shows: (1) TrxG subunits are bound to the genome mostly as

\footnotetext{
(See figure on next page.)

Fig. 5 Distribution of TrxG components in erythroid cells. a The a-globin locus, with shaded areas indicating CGls (green) and putative regulatory regions (blue). ChIP signal names indicated to left. Pileups are shown scaled to $1 \times$ genome coverage, with full scale $0-50 \times$ depth. $\mathbf{b}$ Heatmaps showing normalised/input-subtracted erythroid signals for CFP1 and other TrxG subunits. ChIP indicated directly above each heatmap, and TrxG complex indicated above that. Upper, TSSs; middle, putative enhancers; and lower, CGls (ordered by CGl length). c Colocalisation of subunits of SET1A methyltransferase complexes in a high-confidence peak-set. Light blue and light red regions represent regions with CFP1 and SET1A peaks, respectively. Red outline represents colocalisation between CFP1 and SET1A. Blue outline represents CFP1 peaks colocalised with RBBP5. Number in bold represents overlap of all four SET1A complex subunits (CFP1, SET1A, HCF1, and RBBP5). $d$ Specialisation of TrxG complexes for enhancers and TSSs. "Other" regions are regions containing peaks of at least two TrXG complex subunits that colocalised neither to TSSs nor putative enhancers defined earlier in the study. SET1A/B complexes are represented by CFP1/SET1A, MLL1/2 complexes by MENIN and MLL3/4 by UTX. Note: error bars are derived by assuming that similarly prepared ChIP experiments give a number of peaks governed by Poisson statistics
} 

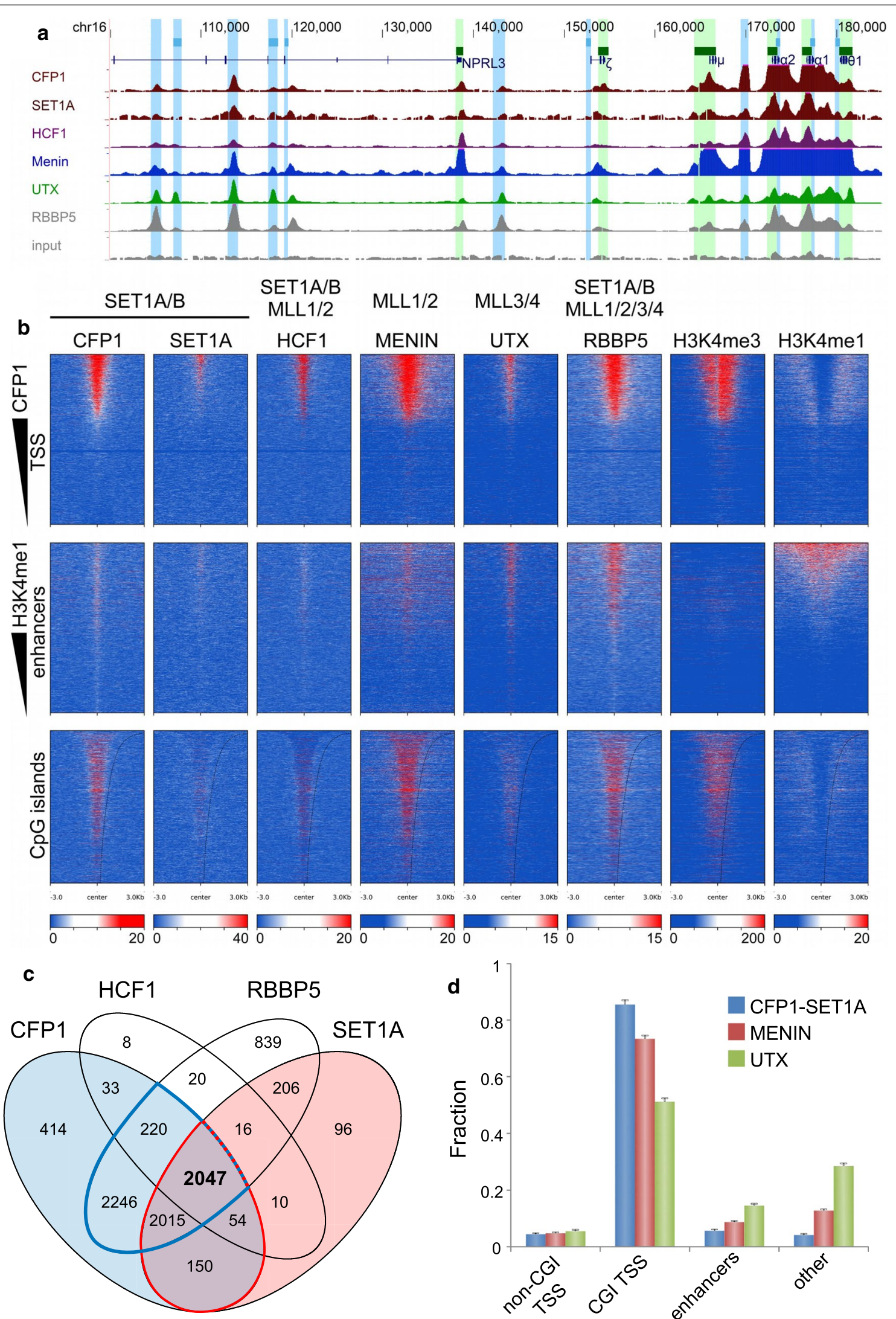
multi-protein complexes, and it is mostly the presence of these complexes that gives rise to the observed ChIP signals; (2) although components of all TrxG complexes may be found at TSSs, CFP1-SET1A complexes are specialised to TSSs relative to other TrxG complexes; and (3) TrxG complex subunits whose functions include establishment of H3K4me3 at bivalent domains and clearing of the repressive H3K27me3 mark are recruited to sites that do not include CFP1.

\section{CFP1 occupancy is associated with specific (epi)genomic features and sequence motifs}

Given the specificity with which CFP1/SET1A complexes bind in the genome, we next asked what sequence-level determinants might account for this. Therefore, CFP1 peaks were analysed for motif enrichment using homer [42], and ten optimised motifs, five from CGI-associated CFP1 peaks and five from non-CGI peaks, were identified (Additional file 1: Table S3, see "Methods"). Four of five motifs derived from non-CGI peaks lacked a CpG dinucleotide, and four of these five motifs were also strongly biased or exclusive for either cell type, whereas the fifth was of low complexity (Additional file 1: Table S3); these results demonstrate that CFP1 is binding in a cell typespecific manner in non-CGI regions. On the other hand, all five motifs optimised in CGI-associated CFP1 peaks contained a CPG dinucleotide and were found at high frequency in CFP1 peak-sets in both EBV and ERY cell types (Additional file 1: Table S3). Genome-wide however, an average of only $4 \%$ of these five CGI-associated motifs (ranging from 2.9 to $6.4 \%$ ) fell within CGIs, similar to the genomic fraction of CpGs (7.0\%) falling within CGIs. This result emphasises the known importance of the CpG dinucleotide, which is bound by CFP1 through its ZF-CxxC domain [17].

To develop a global model of the features specifying CFP1 genomic occupancy, we then analysed the correlation of CFP1 ChIP signal with a number of genomic signals in addition to genomic CPG density in erythroid cells. We considered: (1) that CFP1 is also known to bind all methylation states of H3K4 through its PHD finger domain [26, 33]; (2) that SET1 proteins have been shown to enhance CFP1 interactions with DNA [17]; and (3) that HCF1 and RBBP5 are additional subunits of this complex $[12,36]$. These ChIP intensities and CpG density were averaged in 2-kb windows centred on TSSs and putative enhancers (Figs. 1a, 4 and $5 ; n=41,167$ nonredundant loci). To test the sufficiency of these features to explain CFP1 binding, we computed the correlation of each feature with CFP1 individually (Additional file 1: Table S4). Across all 41,167 TSSs and enhancers (irrespective of chromatin state), all features except H3K4me1 were correlated with the CFP1 signal (Pearson $R^{2}>0.1$,
Additional file 1: Table S4). However, restricting our calculations to TSSs and enhancers having top 10\% chromatin accessibility scores (4117 sites, Additional file 1: Fig. S8, see "Methods"), only three features were still highly correlated with the CFP1 signal: H3K4me3, SET1A and CpG density. Individually, these features could explain 28.6, 16.0 and $15.2 \%$ of the CFP1 signal, respectively, but incorporated in a linear regression model, these features accounted for $47.7 \%$ of CFP1 signal (Additional file 1: Table S5, Additional file 1: Fig. S9). This result shows that these three features contribute mostly additively to CFP1 binding in accessible chromatin regions.

\section{Discussion}

Previous studies have demonstrated a key role for CFP1 in the differentiation of mouse ES cells [22], murine early development [21] and haematopoiesis in mouse and fish [23-25]. Ablation of CFP1 reduces H3K4me3 at CGIs, especially downstream of the TSS, with only moderate changes in gene expression $[15,17]$. Nevertheless, CFP1 lacking DNA-binding properties can target regions with pre-existing low levels of H3K4me3 [15]. Interaction of CFP1 with H3K4me3 occurs via its PHD domain [17, 26]. Here we have studied the genome-wide occupancy of CFP1 in two human haematopoietic lineages. In agreement with a recent genome-wide study in mouse ES cells [17], we found CFP1 associated with transcription from CGI promoters in human haematopoietic lineages, with CFP1 prominently occupying TSSs of both housekeeping and tissue-specific genes.

Several features have been found to be correlated with CFP1 binding to DNA or chromatin. Some of the earlier analyses pointed to the ZF-CXXC domain and its role in binding to CpG dinucleotides [14, 16, 43]. A more recent study has shown a role for SET1A and methylated H3K4 in addition to unmethylated CpGs [17]. Our analysis of these and other (epi)genomic features found that CpG density, H3K4me3 intensity and SET1A intensity were the most prominently correlated with CFP1 occupancy and are sufficient to explain approximately $48 \%$ of CFP 1 binding in open chromatin. This result is particularly compelling in view of expected variability in ChIP-seq signals and implies that CFP1 genomic occupancy is largely determined by: (1) binding of its ZF-CxxC domain to CpG dinucleotides; (2) interaction of its PHD finger domain with methylated forms of H3K4; and (3) stabilisation of binding by interaction with SET1. This multifaceted model of CFP1 binding may explain the association of CFP1 with expression in both the $\alpha$ - and $\beta$-globin TSSs, which differ strongly in CpG density. In agreement with the observations of others [35], this model can explain the tight association we found between ChIP peaks of SET1A/B complex members in high-confidence 
regions of strong CFP1, SET1A, HCF1, Menin, UTX or RBBP5 ChIP peaks.

Focusing on enhancers, we found specific occupancy of CFP1 at enhancers in both cell types together with positive correlation with chromatin accessibility. Moreover, enrichment of SET1A and its strong correlation with CFP1, including at enhancers, support the stabilisation of CFP1 at enhancers. SET1A is known to interact with CFP1 and immobilise CFP1 to DNA [17]. Weaker correlation between CFP1 and chromatin accessibility in enhancers compared to TSSs could be explained by reduced affinity of CFP1 for H3K4me1 compared with H3K4me3 $[17,33]$ and depletion of CGIs in most enhancers.

In addition to the understanding of the mechanisms by which CFP1-containing complexes bind to open chromatin in steady state, an enduring question is how transcription is dynamically regulated in a cell type-specific manner. Early genome-wide studies showed monovalent $\mathrm{H} 3 \mathrm{~K} 27 \mathrm{me} 3$ is a mark of PcG binding at TSSs and is associated with silencing, whereas monovalent H3K4me3 at TSSs is associated with high expression in mouse ES cells. However, TSSs of genes poised for expression are found in an intermediate bivalent (H3K27me3/H3K4me3) state, which arises as a result of targeted H3K4 methyltransferase activity [44-49]. Mutual exclusivity of repressive H3K27me3 and CFP1 genome-wide, as reported here, thus rules out CFP1-SET1 as the methyltransferase complex responsible for the transition of TSSs from repressed to bivalent. Instead, recent evidence shows that MLL2 methyltransferase activity is required for initial trimethylation establishing bivalent promoters in ES cells, whereas MLL1 is at least in part redundant [27, 28].

In differentiated cells, CFP1-SET1 complexes have been found by others to be responsible for most of the H3K4me3 deposition at CGI [16]. In that light, it is interesting that we observed colocalisation between CFP1SET1A and Menin (a component of MLL2 complex). Perhaps even more enlightening is our observation of substantial colocalisation between Menin, RBBP5 (a component of all TrxG complexes) and UTX, suggesting the possibility of frequent co-recruitment of MLL2 and UTX at promoters and enhancers lacking CFP1-SET1A. Recent evidence also suggests the existence of a complex involving MLL2 and UTX [40]. UTX is an H3K27me3 demethylase at target genes $[41,50,51]$ and at poised enhancers as part of MLL3/4 complexes [35, 40,52] and also as a chromatin-remodelling factor [53]. Accordingly, our results suggest that before formation of CFP1-SET1 complexes at TSSs, UTX is recruited together with MLL2 to remove repressive $\mathrm{H} 3 \mathrm{~K} 27 \mathrm{me} 3$ and deposit initial H3K4me3 $[27,28]$. This initial deposition of H3K4me3 by MLL2 complexes could provide an anchor for subsequent binding of CFP1-SET1 complexes, which may colocalise with MLL2 to maintain high levels of H3K4me3, irrespective of CpG content. This model presents a coherent explanation for the transition from repressed to open chromatin in keeping with our observed mutual exclusivity of the H3K27me3 mark and CFP1 binding. This model also places into context the evidence that in differentiated cells methyltransferase activity in bivalent domains switches from MLL2 to SET1A [54] and thus helps to broaden our view of key elements involved in gene regulation through chromatin modification. Further analyses of mutations in key proteins in this regulatory network will be necessary to expand on mechanistic insights into CFP1 functions. These might reveal CFP1 trafficking, protein-protein interactions and their timing, and molecular stoichiometry involved in CFP1-dependent gene regulation. Such follow-up studies might also address the relationship between genomic CFP1 distribution, genome-wide transcriptional states and RNA abundance.

\section{Conclusions}

In view of its known specificity for binding to unmethylated CpG dinucleotides, this study revealed an unexpected association of CFP1 with expressed TSSs, even in the absence of CGIs. Furthermore, CFP1 was associated with Pol II binding at expressed TSSs, regardless of CGI status, and this was observed in both cell types. Intriguingly, in addition to strong occupancy at TSSs, CFP1SET1A complexes were also found at other accessible chromatin regions, including putative enhancers, which were highly enriched in weaker CFP1 peaks. Colocalisation of subunits of other TrxG complexes with CFP1SET1A complexes was also observed in both TSSs and enhancers, though with differential preference, suggesting partial redundancy of TrxG complexes in agreement with the observations of others in pluripotent stem cells [27].

\section{Methods \\ Primary cells}

Primary erythroid cells and newly generated EBVinfected B-lymphoblasts were obtained as previously described [55]. Public EBV ChIP data sets are from the human EBV-infected B lymphocyte cell line GM12878.

\section{ChIP assay}

ChIP was performed as previously described [56]. For CFP1, HCF1, RbBP5, hSET1 (SETD1A), Menin and UTX, chromatin was first cross-linked with ethylene glycol bis(succinimidyl succinate) (EGS) [29] in PBS at a final concentration of $2 \mathrm{mM}$ for $60 \mathrm{~min}$ at RT. Formaldehyde $\left(\mathrm{CH}_{2} \mathrm{O}\right)$ was then added at a final concentration 
of $1 \%$ for $15 \mathrm{~min}$ at RT and samples were sonicated over $20 \min (10 \times 30$-second episodes $)$ at $4{ }^{\circ} \mathrm{C}$ to cleave genomic DNA (Bioruptor, Diagenode). Input data sets were matched with ChIP-seq samples. Antibodies used are: H3K27me3 (07-449) (Millipore); HCF1 (A301400A), RbBP5 (A300-109A), hSET1 (SETD1A, A300289A), Menin (A300-105A), UTX (A302-374A) from Bethyl Labs and CFP1 (CGBP, ab56035) from Abcam. The non-commercial CFP1 antibody was kindly provided by Prof. Robert Roeder. Real-Time PCR was performed using primers and probes (5'FAM-3'TAMRA) for the murine and human $\alpha$-globin locus described previously $[57,58]$. Each ChIP was performed as two independent experiments and quality was assessed by qPCR. Libraries and sequencing (ChIP-seq) were performed using the standard Illumina kits and protocols.

\section{DNasel assay and DNasel sequencing}

Nuclei from human primary erythroid cells were digested with increasing concentrations of DNasel (Roche) as previously described [59]. DNA $(1.5 \mu \mathrm{g})$ from the mid-phase digestions was blunt-ended with T4 DNA Polymerase (NEB) and prepared for Illumina HiSeq 2500 sequencing.

\section{Data sources, protocols and analysis}

Sources of ChIP-seq data are shown in Table 1.

\section{ChIP-Seq analysis}

Reads were aligned using the hisat2 aligner version 2.0.3 with the --no-spliced-alignment option, but otherwise default parameters. Reads were aligned to a splicing-unaware index of the human GRCh38 genome. ChIP peaks were identified using the ENCODE tool Phantompeakqualtools [67], an R script that called peaks using the SPP library [68] version 1.14. Peaks in each experiment were

Table 1 Experiments, protocols, read counts and GEO accession numbers

\begin{tabular}{|c|c|c|c|c|}
\hline Cell type & Data set & Crosslinking/Reference & Read count (paired) & GEO accession \\
\hline Cell type & Data set & Crosslinking/Reference & Read count (paired) & GEO accession \\
\hline Erythroblasts & CFP1 & $\mathrm{EGS}+\mathrm{CH}_{2} \mathrm{O}$ & $38,713,222$ unpaired & GSE114084 \\
\hline Erythroblasts & CFP1 (Abcam) & $\mathrm{EGS}+\mathrm{CH}_{2} \mathrm{O}$ & $38,789,474$ unpaired & GSE114084 \\
\hline Erythroblasts & $\mathrm{H} 3 \mathrm{~K} 27 \mathrm{ac}$ & {$[60]$} & $54,716,349$ unpaired & GSE70660 \\
\hline Erythroblasts & H3K27me3 & $\mathrm{EGS}+\mathrm{CH}_{2} \mathrm{O}$ & $163,108,458$ paired & GSE114084 \\
\hline Erythroblasts & H3K4me1 & {$[60]$} & 26,492,230 unpaired & GSE70660 \\
\hline Erythroblasts & H3K4me3 & {$[61]$} & $14,926,093$ unpaired & GSE36985 \\
\hline Erythroblasts & DNase-seq & $\mathrm{N} / \mathrm{A}$ & 281,771,876 paired & GSE114084 \\
\hline Erythroblasts & ATAC-seq & {$[62]$} & 228,886,766 paired & GSE74912 \\
\hline Erythroblasts & Pol II & {$[61]$} & $106,164,823$ unpaired & GSE36985 \\
\hline Erythroblasts & HCF1 & $\mathrm{EGS}+\mathrm{CH}_{2} \mathrm{O}$ & 179,476,544 paired & GSE114084 \\
\hline Erythroblasts & MEN1 & $\mathrm{EGS}+\mathrm{CH}_{2} \mathrm{O}$ & $81,145,272$ paired & GSE114084 \\
\hline Erythroblasts & RBBP5 & $\mathrm{EGS}+\mathrm{CH}_{2} \mathrm{O}$ & $90,587,516$ paired & GSE114084 \\
\hline Erythroblasts & SET1A & $\mathrm{EGS}+\mathrm{CH}_{2} \mathrm{O}$ & $93,079,696$ paired & GSE114084 \\
\hline Erythroblasts & UTX & $\mathrm{EGS}+\mathrm{CH}_{2} \mathrm{O}$ & $65,366,066$ paired & GSE114084 \\
\hline Erythroblasts, default input data set & Input 1 & $\mathrm{EGS}+\mathrm{CH}_{2} \mathrm{O}$ & 24,173,450 paired & GSE114084 \\
\hline Erythroblasts, matched with HCF1, SET1A & Input 2 & $\mathrm{EGS}+\mathrm{CH}_{2} \mathrm{O}$ & $86,858,360$ paired & GSE114084 \\
\hline Erythroblasts, matched with MEN1 & Input 3 & $\mathrm{EGS}+\mathrm{CH}_{2} \mathrm{O}$ & $109,489,716$ paired & GSE114084 \\
\hline EBV-transformed B cells & CFP1 & $\mathrm{EGS}+\mathrm{CH}_{2} \mathrm{O}$ & 39,009,169 unpaired & GSE114084 \\
\hline EBV-transformed B cells & CFP1 (Abcam) & $\mathrm{EGS}+\mathrm{CH}_{2} \mathrm{O}$ & 41,374,998 unpaired & GSE114084 \\
\hline GM12878 & $\mathrm{H} 3 \mathrm{~K} 27 \mathrm{ac}$ & {$[63]$} & 463,073,456 paired & GSE50893 \\
\hline GM12878 & H3K27me3 & {$[63]$} & 480,207,766 paired & GSE50893 \\
\hline GM12878 & H3K4me1 & {$[63]$} & 271,241,104 paired & GSE50893 \\
\hline GM12878 & H3K4me3 & {$[63]$} & 268,581,398 paired & GSE50893 \\
\hline GM12878 & DNase-seq & {$[64]$} & 400,610,386 unpaired & GSE32970 \\
\hline GM12878 & ATAC-seq & $\begin{array}{l}\text { [65]; first } 24 \mathrm{M} \text { read pairs in SRA } \\
\text { data sets SRR3336945-52 }\end{array}$ & 192,000,000 paired & GSE79921 \\
\hline GM12878 & Pol II & {$[66]$} & $60,061,473$ unpaired & GSE19486 \\
\hline EBV-transformed B cells, default input data set & Input 1 & $\mathrm{EGS}+\mathrm{CH}_{2} \mathrm{O}$ & 19,753,388 paired & GSE114084 \\
\hline GM12878, used with H3K27me3 & Input 2 & [63] & $112,326,958$ paired & GSE50893 \\
\hline
\end{tabular}


thresholded by FDR $<0.01$, except for H3K27me3 in EBV cells, in which case peaks were thresholded by FDR $<0.1$. Narrow peaks were used for analysis of all ChIPs except the H3K27me3 mark, in which case region peaks were analysed. As a confirmation in specific cases, ChIP peaks were secondarily identified using the callpeak function of MACS2, version 2.1.1.20,160,309, with narrow peaks called using a q-value threshold at 0.01 , and broad peaks with the --broad-cutoff 0.1 option. Peak colocalisation analysis was carried out using the ChIPpeakAnno package in $\mathrm{R}$, with maximum gap of $1 \mathrm{~kb}$. General genome arithmetic, including peak intersection with TSSs, was carried out using bedtools [69]. ChIP heatmap plots were generated using the bamCompare, computeMatrix and plotHeatmap functions of deepTools version 2.5.4 [70]. Read depths were compared between ChIP-seq and input data sets using bamCompare with flags --scaleFactorsMethod readCount --ratio subtract --binSize 50 --normalizeTo1x 3100000000 --minMappingQuality 30; this normalisation and subtraction was performed on all data sets. To compare $\mathrm{CpG}$ content signal with ChIP signals, numbers of CpG sites were counted in 50-bp windows, such that 5 CpG sites was computed as $10 \%$ of sites in a 50-bp window. To analyse correlation of two ChIP signals or CpG density and ChIP signals, the given signal distributions were first averaged within 2-kb loci and correlation was computed among loci.

\section{Identification of a set of putative enhancers}

Putative genomic enhancers were identified by a stepwise procedure using Assay for Transposase-Accessible Chromatin (ATAC), Pol II and H3K4me1 signals. First, normalised genome-wide signals for each ChIP were constructed by normalising each pileup to $1 \mathrm{x}$ genome coverage, and subtracting similarly normalised input data using the deepTools bamCompare program as described above. Genomic regions $>2 \mathrm{~kb}$ from gene TSSs exhibiting ATAC-seq signal value $>10$ that also exhibited a Pol II signal value $>5$ were accepted as putative enhancers and confirmed by plotting heatmaps of H3K4me1 and H3K27ac around these loci.

\section{RNA-seq analysis and identification of housekeeping and cell type-specific genes}

RNA-seq data from NCBI GEO (accession GSE74246, three erythroblast samples, and GSE88627, four GM12878 EBV-lymphoblast samples) were aligned to a special purpose, splice-aware version of the GRCh38 human genome downloaded from the hisat2 web page (https://ccb.jhu.edu/software/hisat2/index.shtml). The hisat2 aligner, version 2.0.5, was used with default parameters. Analysis of expression was performed in R: read counts for each locus were obtained in unpaired mode using the featureCounts function from the Rsubread package, and reads per kilobase of transcript per million mapped reads (RPKM) values were calculated using the rpkm function from the edgeR package.

Housekeeping genes and candidate tissue-specific genes were identified from processed RNA-seq expression data (16 tissues) warehoused by the EBI Illumina body map resource (NCBI GEO accession GSE30611). Gene RPKM values were downloaded and thresholded at 0.1. A previously reported method [71] developed for identification of housekeeping exons was applied to whole genes to identify a list of housekeeping genes. Briefly, genes with $\mathrm{RPKM} \geq 0.1$ in all tissues, standard deviation in $\log _{2}(\mathrm{RPKM})<1$, and no tissue with absolute $\log _{2}(\mathrm{RPKM} /$ mean $(\mathrm{RPKM}))>2$ (all expression ratios less than 4 in either direction) were termed housekeeping genes. Genes for which either all expression RPKM $\leq 0.1$ or for which more than half of the tissues had RPKM values $\leq 10 \%$ maximum were considered to be normally non-expressed. The maximal expression RPKM value of these candidate tissue-specific genes was compared to expression RPKM values (averaged across samples) from the analysis of erythroid and lymphoid cell types (above). If a gene was on the list of normally non-expressed genes and its RPKM expression value in erythroid or lymphoid tissue was at least half of this maximum, it was termed tissue-specific in that tissue.

\section{Analysis of TrxG subunit occupancy in a high-confidence subset of peak regions}

To analyse which subunits of TrxG-related methyltransferase complexes colocalised, we compared six ChIP data sets in erythroid cells: CFP1 and SET1A, subunits of SET1A/B complexes; HCF1, a subunit of SET1A/B and MLL1/2 complexes; Menin, a subunit of MLL1/2 complexes; UTX, a subunit of MLL3/4 complexes; and RBBP5, a core subunit of all the complexes. A high-confidence set of peaks in each data set was defined as its most highly enriched tenth percentile, as detected by SPP. Bedtools was used to merge these regions into a non-redundant set of high-confidence peak regions. Subsequently peaks of all strengths detected by SPP were analysed for the presence or absence in these high-confidence regions.

\section{Analysis of motifs in ChIP-enriched regions}

ChIP-enriched regions, identified as peaks by SPP, were analysed for motif enrichment using the homer program [42]. For each ChIP experiment, peaks were divided into two groups by the presence or absence of CGIs. The top 1000 peaks by coverage depth were analysed in each group. Homer selected genomic background sequences matched to target sequences by length and 3-bp oligo content. Homer then used binomial statistics to compare 
motif frequencies in target and background sequences. It first identified enrichments of 364 known motifs, then identified 75 additional de novo motifs ( 25 each at sizes of 8,10 and 12 base pairs). Thus, a total of 300 partially redundant motifs were identified, 150 from CGI-associated CFP1 peaks in both cell types and 150 from nonCGI peaks.

Motifs passing a detection threshold of $p<10^{-20}$ were selected and further optimised by homer, with optimisation carried out separately for CGI and non-CGI peaks. Target sequences used for the optimisation step were combined peaks from ERY and EBV cell types, but with redundant peaks removed. The optimised motifs were clustered by homer and the most significant exemplar in each cluster was reported. The top ten exemplar motifs, five identified from CGI-associated CFP1 peaks and five from non-CGI peaks, were subsequently detected in CGI and non-CGI peaks, respectively. The purpose of this detection was to determine if a given motif, which had been optimised using sequences from both ERY and EBV cells, was biased for ERY or EBV cells; this might occur if the motif reflected genes expressed in that cell type rather than structural binding preference of CFP1.

\section{Analysis of correlation of ChIP signal intensities with (epi) genomic features}

We sought to understand the relationship between the intensity of ChIP and other signals, which might indicate functional interaction. $\mathrm{CpG}$ density and erythroid ChIP signals from CFP1, SET1A, HCF1, RBBP5, H3K4me3, H3K4me1, H3K27me3, DNase-seq and ATAC-seq were analysed, and ChIP signals were $1 \mathrm{x}$-normalised and input was subtracted, as described above. Given that normalised and input-subtracted ChIP signals were noisy, each ChIP signal was averaged in a $2-\mathrm{kb}$ region surrounding each TSS and putative enhancer to give a robust representation of the signal strength. The rare case where robust but non-overlapping ChIP-seq peaks are present at the same locus in different ChIP experiments, which would give a spurious apparent colocalisation, was ignored in this analysis. Relative chromatin accessibility was estimated from DNase-seq and ATAC-seq signals. Given that these signals give differing representations of chromatin accessibility, they were combined; first these signals were linearly scaled by the $\mathrm{z}$-score transformation, and then they were averaged. The resulting chromatin accessibility score was therefore more robust and gave equal weight to DNase-seq and ATAC-seq data.

Tests of Pearson's product moment correlation $\left(R^{2}\right)$ were performed between CFP1 and CpG density, SET1A, HCF1, RBBP5, H3K4me1, H3K4me3 and chromatin accessibility; this was done in all loci and in a subset of loci with upper decile chromatin accessibility scores.
Features whose correlation $R^{2}$ value was greater than $10 \%$ were identified. To test the sufficiency of these features to explain CFP1 occupancy in open chromatin, a linear regression analysis was performed, and this analysis was limited to the top-decile accessible regions.

\section{Additional file}

Additional file 1. Fig. S1: Analysis of CFP1 binding at individual loci and CpG islands (CGIs). (A-B) Analysis of CFP1 binding at the human a-globin locus in expressing and non-expressing cells. (A) Real-Time PCR analysis of immunoprecipitated chromatin using CFP1 antibody in human erythroblasts (red) and B-lymphocytes (blue). The y-axis represents enrichment over the input DNA, normalised to a control sequence in the human 185 gene. The $x$-axis represents the positions of Taqman probes used. The coding sequence is represented by the three exons (Promoter/Ex1, Ex2, Ex3) of the a-globin genes. 218 and hBact denote control sequences adjacent to the CpG islands of the human LUC7L (218) and ACTB promoters. Error bars correspond to \pm 1 SD from at least two independent ChIPs. (B) Real-Time PCR analysis of immunoprecipitated chromatin using the CFP1 antibody indicated in humanised erythroblasts (normal, +MCS-R2 (left) and mutant, MCS-R2 (right). The y-axis represents enrichment over the input DNA, normalised to a control sequence in the mouse GAPDH gene. CpG Act denotes additional control sequence at the CGI of the mouse ACTB gene. The amplicons highlighted in red represent deleted regions in the humanised mice, for which no PCR signal is observed. Error bars correspond to \pm 1 SD from at least two independent ChIPs. (C) CFP1 ChIP signal intensity in the top 200 peaks, by antibody and by cell type. Abcam, ab56035 antibody. Roeder, main antibody used in this study. (D) Analysis of CGI (green) and non-CGl (blue) transcription start sites (1-kb window, centred on TSS). Gene symbols shown with CpG content of individual loci in parentheses. Greek letters represent individual globin genes. Fig. S2: Peak overlaps of CFP1 and marks of active and repressed chromatin in transcription start sites (TSSs). Peaks were detected by MACS2. Venn diagrams show that CFP1 peaks within 1-kb of TSSs are strongly associated with $\mathrm{H} 3 \mathrm{~K} 4 \mathrm{me} 3$ histone mark and poorly associated with $\mathrm{H} 3 \mathrm{~K} 27$ me3 repressive histone mark. Cell types are (A) ERY and (B) EBV. Public data sets: * NCBI GEO GSE36985, ** NCBI GEO GSE50893. Fig. S3: UCSC tracks showing CFP1 and other ChIP signals in gene loci in erythroblasts (ERY) and EBV-transformed B-lymphoblasts (EBV). Hg38 coordinates for multiple genes, CpG islands (CGl, green boxes), and putative regulatory regions (blue boxes) are shown. CFP1 signals are shown in dark reds, inputs in grey, histone $\mathrm{H} 3$ signals in blues and open chromatin marks in greens. All ChIP pileups are scaled to $1 \times$ coverage genome-wide and shown in a range $0-50$, except CFP1 (Roeder) is shown with extended range and H3K27me3 graphs scaled by 2x. (A) Tissue-specific binding of CFP1 to CGI promoters of tissue-specifically expressed genes. Left (chr16), CGI promoters of active genes in alpha globin locus are CFP1-bound in ERY, and unbound in EBV. Flanking regions are included, with known tissuespecific enhancers. Right (chr6), first seven exons of IRF4 locus, active in EBV and inactive in ERY, with CFP1 binding to CGI promoter in EBV only. (B) CGI promoters of housekeeping genes are CFP1 bound and unmarked by H3K27me3. Left (chr7), ACTB locus. Right (chr16), LUC7L locus. (C) CGI promoter of RHBDF1 locus (chr16) has H3K27me3 mark and the absence of CFP1 binding in both ERY and EBV. Fig. S4: Western blot analysis of CGBP (CFP1) expression in mouse and human erythroid and human lymphoid cell types. Whole cell extracts $(20 \mu \mathrm{g})$ were loaded in each lane (1) mouse ES, (2) U-MEL, (3) I-MEL, (4) mouse primary erythroblasts and (5) human primary $T$ lymphocytes and (6) human primary erythroblasts and separated on a 10\% SDS-polyacrylamide gel. CFP1 antibody was used at a 1:1000 dilution. Fig. S5: Similar cell type-specific CFP1 read depth at CGl TSS of HBA1 gene and non-CGITSS of HBB gene. Upper two tracks use the main antibody, and second two tracks use the commercial antibody. Coordinates are from the hg38 human genome build. Read depths are averaged in $50 \mathrm{bp}$ bins and normalised to $1 \times$ genome-wide coverage. 
Blue boxes, known regulatory regions; green box, CGI. Fig. S6: Distribution of TrxG components in erythroid cells. Green indicates CGI and blue indicates other putative regulatory regions. All loci transcribed right to left. Pileups are shown scaled to $1 \times$ genome coverage, with full scale $0-50 x$ depth. (A) Housekeeping genes ACTB, left (chr7), and LUC7L, right (chr16). (B) $\beta$-globin locus (chr11), (C) Non-expressed RHBDF1 locus (chr16). Fig. S7: Overlap of TrxG subunit ChIP peaks in a high-confidence subset of regions. SET1A complexes are represented by CFP1-SET1A colocalisation. MLL1/2 complexes are represented by Menin, and MLL3/4 complexes are represented by UTX, respectively. HCF1 is found in SET1A/B and MLL1/2 complexes, and RBBP5 is a member of SET1A/B and MLL1/2/3/4 complexes. Red outline (4220 peaks) shows strong colocalisation of Menin and CFP1-SET1A, accounting for the vast majority (99.5\%) of 4242 CFP1-SET1A and half (50.0\%) of 8432 Menin peak regions. Majority $(87.0 \%$, 2089/2400 peaks) of HCF1 (blue region) is accounted for by approximately half $(49.5 \%, 2089 / 4220)$ of regions of Menin-SET1A-CFP1 colocalisation. Regions where either SET1A-CFP1 or Menin or both are colocalised with HCF1 (blue dashed line) accounts for nearly all $(99.6 \%, 2390 / 2400)$ HCF1 regions, suggesting that HCF1 bound to DNA is primarily present as part of SET1A/B or MLL1/2 complexes. Fig. S8: Chromatin accessibility in TSSs and enhancers in erythroid cells as measured by ATAC-seq and DNaseseq. 1X-normalised, input-subtracted signals from ATAC-seq and DNase were averaged in a $2-\mathrm{kb}$ window about TSSs and putative enhancers. Z-score transformed values for ATAC-seq and DNase-seq at a given locus were averaged. Fig. S9: Relationship of CFP1 signal to three predictive factors in top-decile open chromatin regions. A linear combination of $\mathrm{CpG}$ density and SET1A and H3K4me3 ChIP signals explains a substantial fraction of variation in CFP1 ChIP signal. Table S1: Bias of CFP1 for CGI TSSs in cell types and gene classes. Table S2: Bias of CFP1 for housekeeping gene TSSs. Table S3: Motifs associated with CFP1 peaks. Table S4: Dependence of CFP1 ChIP signal in erythroid cells on covariates putatively associated with its binding. Table S5: Analysis of variance of CFP1 signal in top-decile open chromatin regions surrounding TSSs and putative enhancers.

\section{Authors' contributions}

LNL did the bioinformatics analysis, interpreted results, methodology, resources and wrote the manuscript; MF did formal bioinformatics analysis; MDL did formal bioinformatics analysis and drafted the manuscript; DG participated in the design of the study and wrote the manuscript; SRT supervised bioinformatics analysis, interpreted results and wrote manuscript; KRK participated in the coordination of the study and drafted the manuscript; DV conceived the study and participated in its design, carried out the molecular studies, interpreted results, coordinated the study and drafted the manuscript. All authors read and approved the final manuscript.

\section{Author details}

${ }^{1}$ The Roslin Institute and Royal (Dick) School of Veterinary Studies, University of Edinburgh, Easter Bush, Midlothian EH25 9RG, UK. ${ }^{2}$ MRC Molecular Haematology Unit, Weatherall Institute for Molecular Medicine, John Radcliffe Hospital, University of Oxford, Oxford OX3 9DS, UK. ${ }^{3}$ Centre for Stem Cells and Regenerative Medicine, 28th Floor Guy's Tower, Great Maze Pond, London SE1 9RT, UK. ${ }^{4}$ INSERM, UMRS-1126, Institut Universitaire d'Hématologie, Université Paris Diderot, 75010 Paris, France. ${ }^{5}$ MRC Centre for Regenerative Medicine, University of Edinburgh, 5 Little France Drive, Edinburgh EH16 4 UU, UK. ${ }^{6}$ Laboratory of Haematopoietic Stem Cell \& Leukaemia Biology, Centre for Haemato-Oncology, Barts Cancer Institute, Queen Mary University of London, Charterhouse Square, London EC1M 6BQ, UK. ${ }^{7}$ st John's institute of dermatology, Great Maze Pond, London SE1 9RT, UK.

\section{Acknowledgements}

We are very grateful to Zhanyun Tang and Bob Roeder for the CFP1 antibody. We would like to thank our colleagues Alan Archibald, Philipp Voigt and Duncan Sproul for critically reading the manuscript. We also thank Jim Hughes for curating data sets obtained in Oxford. High-throughput sequencing was provided by the Oxford Genomics Centre (http://www.well.ox.ac.uk/ogc/ home/) and Edinburgh Genomics (http://genomics.ed.ac.uk).

\section{Competing interests}

The authors declare that they have no competing interests.

\section{Availability of data and materials}

The data sets generated and/or analysed during the current study are available in the NCBI Gene Expression Omnibus repository, GSE114084 [https:// www.ncbi.nlm.nih.gov/geo/query/acc.cgi?acc=GSE114084].

\section{Consent for publication}

All authors read and approved the final manuscript.

\section{Ethics approval and consent to participate}

Study protocols received ethical approval from Oxford University Ethical Review Panel and all animal work was carried out under Home Office License. The data were analysed anonymously.

\section{Funding}

This work was supported by a University of Edinburgh Chancellor's Fellowship to Douglas Vernimmen and by Institute Strategic Grant funding to the Roslin Institute from the BBSRC [BB/J004235/1] and [BB/P013732/1]. Louie N. van de Lagemaat was supported by Roslin Institute funding to Douglas Vernimmen.

\section{Publisher's Note}

Springer Nature remains neutral with regard to jurisdictional claims in published maps and institutional affiliations.

Received: 13 July 2018 Accepted: 28 September 2018

Published online: 06 October 2018

\section{References}

1. Deaton AM, Bird A. CpG islands and the regulation of transcription. Genes Dev. 2011;25(10):1010-22.

2. Illingworth RS, Bird AP. CpG islands-'a rough guide.' FEBS Lett. 2009;583(11):1713-20.

3. Mouse Genome Sequencing C, Waterston RH, Lindblad-Toh K, Birney E, Rogers J, Abril JF, et al. Initial sequencing and comparative analysis of the mouse genome. Nature. 2002;420(6915):520-62.

4. Antequera F. Structure, function and evolution of $\mathrm{CpG}$ island promoters Cell Mol Life Sci. 2003;60(8):1647-58.

5. Lynch MD, Smith AJ, De Gobbi M, Flenley M, Hughes JR, Vernimmen D, et al. An interspecies analysis reveals a key role for unmethylated CpG dinucleotides in vertebrate Polycomb complex recruitment. EMBO J. 2012;31(2):317-29.

6. Antequera F, Bird A. Number of $\mathrm{CpG}$ islands and genes in human and mouse. Proc Natl Acad Sci USA. 1993;90(24):11995-9.

7. Matsuo K, Clay O, Takahashi T, Silke J, Schaffner W. Evidence for erosion of mouse CpG islands during mammalian evolution. Somat Cell Mol Genet. 1993;19(6):543-55.

8. Roberts SA, Gordenin DA. Hypermutation in human cancer genomes: footprints and mechanisms. Nat Rev Cancer. 2014;14(12):786-800.

9. Vernimmen D. Uncovering enhancer functions using the alpha-globin locus. PLoS Genet. 2014;10(10):e1004668.

10. Long HK, Blackledge NP, Klose RJ. ZF-CxxC domain-containing proteins, $\mathrm{CpG}$ islands and the chromatin connection. Biochem Soc Trans. 2013;41(3):727-40.

11. Piunti A, Shilatifard A. Epigenetic balance of gene expression by Polycomb and COMPASS families. Science. 2016;352(6290):aad9780.

12. Howe FS, Fischl H, Murray SC, Mellor J. Is H3K4me3 instructive for transcription activation? BioEssays. 2017;39(1):1-12.

13. Lee JH, Skalnik DG. CpG-binding protein (CXXC finger protein 1) is a component of the mammalian Set1 histone H3-Lys4 methyltransferase complex, the analogue of the yeast Set1/COMPASS complex. J Biol Chem. 2005;280(50):41725-31.

14. Xu C, Bian C, Lam R, Dong A, Min J. The structural basis for selective binding of non-methylated CpG islands by the CFP1 CXXC domain. Nat Commun. 2011;2:227.

15. Clouaire T, Webb S, Skene P, Illingworth R, Kerr A, Andrews R, et al. Cfp 1 integrates both CpG content and gene activity for accurate H3K4me3 deposition in embryonic stem cells. Genes Dev. 2012;26(15):1714-28. 
16. Thomson JP, Skene PJ, Selfridge J, Clouaire T, Guy J, Webb S, et al. CpG islands influence chromatin structure via the $\mathrm{CpG}$-binding protein Cfp1. Nature. 2010;464(7291):1082-6.

17. Brown DA, Di Cerbo V, Feldmann A, Ahn J, Ito S, Blackledge NP, et al. The SET1 complex selects actively transcribed target genes via multivalent interaction with CpG island chromatin. Cell Rep. 2017;20(10):2313-27.

18. Hu D, Gao X, Morgan MA, Herz HM, Smith ER, Shilatifard A. The MLL3/ MLL4 branches of the COMPASS family function as major histone H3K4 monomethylases at enhancers. Mol Cell Biol. 2013;33(23):4745-54.

19. Kaikkonen MU, Spann NJ, Heinz S, Romanoski CE, Allison KA, Stender $J \mathrm{D}$, et al. Remodeling of the enhancer landscape during macrophage activation is coupled to enhancer transcription. Mol Cell. 2013;51(3):310-25.

20. Lee JE, Wang C, Xu S, Cho YW, Wang L, Feng X, et al. H3K4 mono- and di-methyltransferase MLL4 is required for enhancer activation during cell differentiation. Elife. 2013;2:e01503.

21. Carlone DL, Skalnik DG. CpG binding protein is crucial for early embryonic development. Mol Cell Biol. 2001;21(22):7601-6.

22. Carlone DL, Lee JH, Young SR, Dobrota E, Butler JS, Ruiz J, et al. Reduced genomic cytosine methylation and defective cellular differentiation in embryonic stem cells lacking CpG binding protein. Mol Cell Biol. 2005;25(12):4881-91.

23. Chun KT, Li B, Dobrota E, Tate C, Lee JH, Khan S, et al. The epigenetic regulator $\mathrm{CXXC}$ finger protein 1 is essential for murine hematopoiesis. PLOS ONE. 2014;9(12):e113745.

24. Huang HT, Kathrein KL, Barton A, Gitlin Z, Huang YH, Ward TP, et al. A network of epigenetic regulators guides developmental haematopoiesis in vivo. Nat Cell Biol. 2013;15(12):1516-25.

25. Young SR, Mumaw C, Marrs JA, Skalnik DG. Antisense targeting of CXXC finger protein 1 inhibits genomic cytosine methylation and primitive hematopoiesis in zebrafish. J Biol Chem. 2006;281 (48):37034-44.

26. Mahadevan J, Skalnik DG. Efficient differentiation of murine embryonic stem cells requires the binding of CXXC finger protein 1 to DNA or methylated histone H3-Lys4. Gene. 2016;594(1):1-9.

27. Denissov S, Hofemeister H, Marks H, Kranz A, Ciotta G, Singh S, et al. Mll2 is required for $\mathrm{H} 3 \mathrm{~K} 4$ trimethylation on bivalent promoters in embryonic stem cells, whereas MII1 is redundant. Development. 2014;141(3):526-37.

28. Hu D, Garruss AS, Gao X, Morgan MA, Cook M, Smith ER, et al. The MII2 branch of the COMPASS family regulates bivalent promoters in mouse embryonic stem cells. Nat Struct Mol Biol. 2013;20(9):1093-7.

29. Vernimmen D, Lynch MD, De Gobbi M, Garrick D, Sharpe JA, SloaneStanley JA, et al. Polycomb eviction as a new distant enhancer function. Genes Dev. 2011;25(15):1583-8.

30. Tang Z, Chen WY, Shimada M, Nguyen UT, Kim J, Sun XJ, et al. SET1 and p300 act synergistically, through coupled histone modifications, in transcriptional activation by p53. Cell. 2013;154(2):297-310.

31. Wallace HA, Marques-Kranc F, Richardson M, Luna-Crespo F, Sharpe $J A$, Hughes J, et al. Manipulating the mouse genome to engineer precise functional syntenic replacements with human sequence. Cell. 2007;128(1):197-209.

32. Vernimmen D, Marques-Kranc F, Sharpe JA, Sloane-Stanley JA, Wood WG, Wallace HA, et al. Chromosome looping at the human alpha-globin locus is mediated via the major upstream regulatory element (HS -40). Blood. 2009;114(19):4253-60.

33. Eberl HC, Spruijt CG, Kelstrup CD, Vermeulen M, Mann M. A map of general and specialized chromatin readers in mouse tissues generated by label-free interaction proteomics. Mol Cell. 2013;49(2):368-78.

34. Mokry M, Hatzis P, Schuijers J, Lansu N, Ruzius FP, Clevers H, et al. Integrated genome-wide analysis of transcription factor occupancy, RNA polymerase II binding and steady-state RNA levels identify differentially regulated functional gene classes. Nucleic Acids Res. 2012;40(1):148-58

35. van Nuland R, Smits AH, Pallaki P, Jansen PW, Vermeulen $M$, Timmers $\mathrm{HT}$. Quantitative dissection and stoichiometry determination of the human SET1/MLL histone methyltransferase complexes. Mol Cell Biol. 2013:33(10):2067-77.

36. Li Y, Han J, Zhang Y, Cao F, Liu Z, Li S, et al. Structural basis for activity regulation of MLL family methyltransferases. Nature. 2016;530(7591):447-52.

37. Jenuwein T, Laible G, Dorn R, Reuter G. SET domain proteins modulate chromatin domains in eu- and heterochromatin. Cell Mol Life Sci. 1998;54(1):80-93.
38. Lee JH, Tate CM, You JS, Skalnik DG. Identification and characterization of the human Set1B histone H3-Lys4 methyltransferase complex. J Biol Chem. 2007;282(18):13419-28.

39. Mellor J, Woloszczuk R, Howe FS. The Interleaved Genome. Trends Genet. 2016:32(1):57-71.

40. Issaeva I, Zonis Y, Rozovskaia T, Orlovsky K, Croce CM, Nakamura T, et al. Knockdown of ALR (MLL2) reveals ALR target genes and leads to alterations in cell adhesion and growth. Mol Cell Biol. 2007;27(5):1889-903.

41. Lee HG, Kahn TG, Simcox A, Schwartz YB, Pirrotta V. Genome-wide activities of Polycomb complexes control pervasive transcription. Genome Res. 2015;25(8):1170-81.

42. Heinz S, Benner C, Spann N, Bertolino E, Lin YC, Laslo P, et al. Simple combinations of lineage-determining transcription factors prime cisregulatory elements required for macrophage and B cell identities. Mol Cell. 2010;38(4):576-89.

43. Blackledge NP, Thomson JP, Skene PJ. CpG island chromatin is shaped by recruitment of ZF-CxxC proteins. Cold Spring Harb Perspect Biol. 2013:5(11):a018648

44. Mikkelsen TS, Ku M, Jaffe DB, Issac B, Lieberman E, Giannoukos G, et al. Genome-wide maps of chromatin state in pluripotent and lineage-committed cells. Nature. 2007;448(7153):553-60.

45. Azuara V, Perry P, Sauer S, Spivakov M, Jorgensen HF, John RM, et al. Chromatin signatures of pluripotent cell lines. Nat Cell Biol. 2006;8(5):532-8.

46. Bernstein BE, Mikkelsen TS, Xie X, Kamal M, Huebert DJ, Cuff J, et al. A bivalent chromatin structure marks key developmental genes in embryonic stem cells. Cell. 2006;125(2):315-26.

47. Ku M, Koche RP, Rheinbay E, Mendenhall EM, Endoh M, Mikkelsen TS, et al. Genomewide analysis of PRC1 and PRC2 occupancy identifies two classes of bivalent domains. PLoS Genet. 2008:4(10):e1000242.

48. Pan G, Tian S, Nie J, Yang C, Ruotti V, Wei H, et al. Whole-genome analysis of histone $\mathrm{H} 3$ lysine 4 and lysine 27 methylation in human embryonic stem cells. Cell Stem Cell. 2007;1 (3):299-312.

49. Zhao XD, Han X, Chew JL, Liu J, Chiu KP, Choo A, et al. Whole-genome mapping of histone $\mathrm{H} 3 \mathrm{Lys} 4$ and 27 trimethylations reveals distinct genomic compartments in human embryonic stem cells. Cell Stem Cell. 2007;1(3):286-98.

50. Agger K, Cloos PA, Christensen J, Pasini D, Rose S, Rappsilber J, et al. UTX and JMJD3 are histone H3K27 demethylases involved in HOX gene regulation and development. Nature. 2007;449(7163):731-4.

51. Dhar SS, Lee SH, Chen K, Zhu G, Oh W, Allton K, et al. An essential role for UTX in resolution and activation of bivalent promoters. Nucleic Acids Res. 2016:44(8):3659-74

52. Cho YW, Hong T, Hong S, Guo H, Yu H, Kim D, et al. PTIP associates with MLL3- and MLL4-containing histone H3 lysine 4 methyltransferase complex. J Biol Chem. 2007;282(28):20395-406.

53. Miller SA, Mohn SE, Weinmann AS. Jmjd3 and UTX play a demethylaseindependent role in chromatin remodeling to regulate T-box family member-dependent gene expression. Mol Cell. 2010;40(4):594-605.

54. Sze CC, Cao K, Collings CK, Marshall SA, Rendleman EJ, Ozark PA, et al. Histone H3K4 methylation-dependent and -independent functions of Set1A/COMPASS in embryonic stem cell self-renewal and differentiation. Genes Dev. 2017;31(17):1732-7.

55. Pope SH, Fibach E, Sun J, Chin K, Rodgers GP. Two-phase liquid culture system models normal human adult erythropoiesis at the molecular level. Eur J Haematol. 2000;64(5):292-303.

56. Vernimmen D, De Gobbi M, Sloane-Stanley JA, Wood WG, Higgs DR. Long-range chromosomal interactions regulate the timing of the transition between poised and active gene expression. EMBO J. 2007:26(8):2041-51.

57. Anguita E, Hughes J, Heyworth C, Blobel GA, Wood WG, Higgs DR. Globin gene activation during haemopoiesis is driven by protein complexes nucleated by GATA-1 and GATA-2. EMBO J. 2004;23(14):2841-52.

58. De Gobbi M, Anguita E, Hughes J, Sloane-Stanley JA, Sharpe JA, Koch CM, et al. Tissue-specific histone modification and transcription factor binding in alpha globin gene expression. Blood. 2007;110(13):4503-10.

59. Kowalczyk MS, Hughes JR, Garrick D, Lynch MD, Sharpe JA, Sloane-Stanley JA, et al. Intragenic enhancers act as alternative promoters. Mol Cell. 2012;45(4):447-58

60. Huang J, Liu X, Li D, Shao Z, Cao H, Zhang Y, et al. Dynamic control of enhancer repertoires drives lineage and stage-specific transcription during hematopoiesis. Dev Cell. 2016;36(1):9-23. 
61. Xu J, Shao Z, Glass K, Bauer DE, Pinello L, Van Handel B, et al. Combinatorial assembly of developmental stage-specific enhancers controls gene expression programs during human erythropoiesis. Dev Cell. 2012;23(4):796-811.

62. Corces MR, Buenrostro JD, Wu B, Greenside PG, Chan SM, Koenig JL, et al. Lineage-specific and single-cell chromatin accessibility charts human hematopoiesis and leukemia evolution. Nat Genet. 2016;48(10):1193-203.

63. Kasowski M, Kyriazopoulou-Panagiotopoulou S, Grubert F, Zaugg JB, Kundaje A, Liu Y, et al. Extensive variation in chromatin states across humans. Science. 2013;342(6159):750-2.

64. Thurman RE, Rynes E, Humbert R, Vierstra J, Maurano MT, Haugen E, et al. The accessible chromatin landscape of the human genome. Nature. 2012;489(7414):75-82.

65. Chen X, Shen Y, Draper W, Buenrostro JD, Litzenburger U, Cho SW, et al. ATAC-see reveals the accessible genome by transposase-mediated imaging and sequencing. Nat Methods. 2016;13(12):1013-20.
66. Kasowski M, Grubert F, Heffelfinger C, Hariharan M, Asabere A, Waszak SM, et al. Variation in transcription factor binding among humans. Science. 2010;328(5975):232-5.

67. Marinov GK, Kundaje A, Park PJ, Wold BJ. Large-scale quality analysis of published ChIP-seq data. G3 (Bethesda). 2014;4(2):209-23.

68. Kharchenko PV, Tolstorukov MY, Park PJ. Design and analysis of ChIP-seq experiments for DNA-binding proteins. Nat Biotechnol. 2008;26(12):1351-9.

69. Quinlan AR, Hall IM. BEDTools: a flexible suite of utilities for comparing genomic features. Bioinformatics. 2010;26(6):841-2.

70. Ramirez F, Dundar F, Diehl S, Gruning BA, Manke T. deepTools: a flexible platform for exploring deep-sequencing data. Nucleic Acids Res. 2014;42(Web Server issue):W187-91.

71. Eisenberg E, Levanon EY. Human housekeeping genes, revisited. Trends Genet. 2013;29(10):569-74.
Ready to submit your research? Choose BMC and benefit from:

- fast, convenient online submission

- thorough peer review by experienced researchers in your field

- rapid publication on acceptance

- support for research data, including large and complex data types

- gold Open Access which fosters wider collaboration and increased citations

- maximum visibility for your research: over $100 \mathrm{M}$ website views per year

At BMC, research is always in progress.

Learn more biomedcentral.com/submissions 